\title{
Ruin Theory with Excess of Loss Reinsurance and Reinstatements
}

\author{
Hansjörg Albrecher*, $†$ Sandra Haas ${ }^{\ddagger}$
}

\begin{abstract}
The present paper studies the probability of ruin of an insurer, if excess of loss reinsurance with reinstatements is applied. In the setting of the classical CramerLundberg risk model, piecewise deterministic Markov processes are used to describe the free surplus process in this more general situation. It is shown that the finite-time ruin probability is both the solution of a partial integro-differential equation and the fixed point of a contractive integral operator. We exploit the latter representation to develop and implement a recursive algorithm for numerical approximation of the ruin probability that involves high-dimensional integration. Furthermore we study the behavior of the finite-time ruin probability under various levels of initial surplus and security loadings and compare the efficiency of the numerical algorithm with the computational alternative of stochastic simulation of the risk process.
\end{abstract}

Keywords: reinsurance, piecewise deterministic Markov process, integral operator, finitetime ruin probability, high-dimensional integration

\section{Introduction}

Excess of loss (XL) reinsurance contracts are widely used in insurance practice and many results on optimal reinsurance schemes under different premium principles and objective functions can be found in the literature (for a survey, see for instance [2]). In practice, the reinsurer rarely offers a pure excess of loss contract, but adds clauses, such as limiting his aggregate liability or adding reinstatements. Clauses limiting the aggregate claims of the reinsurer and its effects are well understood from a theoretical perspective. On the other hand, although reinstatements are quite common in practice (in particular for casualty

\footnotetext{
*Corresponding Author.

${ }^{\dagger}$ Department of Actuarial Science, Faculty of Business and Economics, University of Lausanne, Switzerland and Swiss Finance Institute. Tel:+41-21-692-3371, Fax:+41-21-692-3435. email: hansjoerg.albrecher@unil.ch

${ }^{\ddagger}$ Department of Actuarial Science, Faculty of Business and Economics, University of Lausanne, Switzerland. email: sandra.haas@unil.ch
} 
insurance), literature dealing with a rigorous and quantitative approach to the subject is scarce. The main focus of the existing literature is on the calculation of premiums for these contracts under different premium principles, see e.g. Sundt [11] for an early reference. Mata [8] studied the joint distribution for consecutive layers with reinstatements and applied the $\mathrm{PH}$ transform to calculate the premiums for free and paid reinstatements. For the discrete case, Walhin and Paris [13] derived a multivariate Panjer-type algorithm in order to approximate the resulting compound claim distribution for the cedent. A distribution-free approximation in a market with incomplete information was given by Hürlimann [7]. Hess and Schmidt [6] determine an optimal premium plan for reinsurance contracts with reinstatements by minimizing the expected squared difference between the loss and the premium income of the reinsurer. Walhin et al. [12] discussed practical pricing of excess of loss treaties and introduced reinstatements as clauses that make the premiums random.

In terms of effects of reinstatements on the solvency of the cedent, Walhin and Paris [13] calculated the probability of ruin in a discrete-time risk model using recursive methods.

The goal of this paper is to determine the probability of ruin in a continuous-time risk model. In a first step we show that the concept of piecewise deterministic Markov processes (PDMP) allows a suitable Markovian description of the surplus process of the cedent by adding the reinsurance cover as another dimension. PDMP's in an insurance framework were first studied by Dassios and Embrechts [4], see Rolski et al. [9] for an overview.

Since one cannot expect analytical solutions for the resulting complicated dynamics, numerical techniques have to be applied. We formulate a contractive integral operator whose fixed point is the required finite-time ruin probability. Iterative application of this integral operator will lead to a high-dimensional integration problem to approximate the exact solutions. We investigate the feasibility of such an approach and illustrate the procedure with numerical results for exponentially distributed claim sizes and an expected value principle for the calculation of the premiums. Finally, we compare the results with approximations obtained from simulation of the underlying risk process.

\section{XL reinsurance with reinstatements}

Let the independent and identically distributed random variables $X_{i}\left(i=1, \ldots, N_{t}\right)$ denote individual claim sizes in an insurance portfolio, where the random variable $N_{t}$ is the number of claims up to time $t$. $N_{t}$ is assumed to be Poisson distributed with parameter $\lambda$, and $X_{i}$ and $N_{t}$ are independent.

The surplus process of the insurance portfolio at time $t$ in the classical risk model is defined by

$$
R(t)=u_{0}+\beta t-\sum_{i=1}^{N_{t}} X_{i}
$$


where $u_{0}$ is the initial surplus of the cedent and $\beta$ is the gross premium income per time unit.

Let $T_{u}=\inf \{t: R(t)<0\}$ denote the time of ruin and $\psi(u)=\mathbb{P}\left(T_{u}<\infty\right)$ the infinite-time ruin probability. The finite-time ruin probability is denoted by $\psi(u, T)=\mathbb{P}\left(T_{u}<T\right)$.

In a plain XL contract with retention $l$, the reinsurer covers the part of each claim that exceeds $l$. In practice the reinsurer usually limits the covered part by an upper bound $l+m$, such that the reinsurer's part $Z_{i}$ and the cedent's part $C_{i}$, respectively, are

$$
Z_{i}=\left\{\begin{array}{ll}
0, & X_{i} \leq l \\
X_{i}-l, & l \leq X_{i} \leq l+m \\
m, & X_{i} \geq l+m
\end{array} \quad \text { and } \quad C_{i}= \begin{cases}X_{i}, & X_{i} \leq l \\
l, & l \leq X_{i} \leq l+m \\
X_{i}-m, & X_{i} \geq l+m\end{cases}\right.
$$

The aggregate cover of the reinsurer up to time $t$ is then $Z=Z(t)=\sum_{i=1}^{N_{t}} Z_{i}$.

In addition, the reinsurer often limits this aggregate liability to an amount $M$, which usually is an integer multiple of the individual maximum cover, i.e. $M=(k+1) m$. In this case the reinsurer is said to offer $k$ reinstatements.

Under this setting, the reinsurer's part becomes $R_{k}=\min (Z,(k+1) m)$ and the cover of the $j$-th reinstatement is therefore $r_{j}=\min (\max (Z-j m, 0), m)$.

In this paper we assume a pro-rata-capita concept: At the beginning of the XL reinsurance contract the cedent pays an initial premium $p_{0}$ for the reinsurance cover $r_{0}=\min (Z, m)$. If a claim $X_{i}$ occurs which uses up part of the cover, the cover is reinstated and the cedent pays at this moment an additional premium $P_{j}$ to reinstate the used up part of the layer. This premium is a multiple of the fraction of the cover used up of the last reinstatement $r_{j-1}:$

$$
P_{j}=\frac{c p_{0}}{m} \min (\max (Z-(j-1) m, 0), m),
$$

where $c>0$ is a percentage of the initial premium $p_{0}$ (in some contracts, $c$ may depend on $j$ as well).

Therefore it can happen that multiple claims use up one reinstated layer, but also that one claim uses two reinstatements $\left(r_{j^{\prime}-1}\right.$ and $\left.r_{j^{\prime}}\right)$ partly. In this case the charged premium for the reinstatement of the cover is the sum of $P_{j^{\prime}}$ and $P_{j^{\prime}+1}$.

The advantage of such reinstatement clauses for the reinsurer is obviously the limitation of the total reinsurance cover. The advantage for the cedent is that there is less financial burden at the beginning of the contract (as only $p_{0}$ is paid) and in addition, the total reinsurance premium is reduced, as premiums for the further reinstatements are only paid for the actually needed covers.

In the following we give an illustration of an XL contract with reinstatements.

Example 2.1: Assume an XL reinsurance contract with a layer of [100,200] with two additional reinstatements, at $c=0.5$. In the concrete situation assume now that the cedent faces four claims, namely $X_{1}=150, X_{2}=300, X_{3}=175$ and $X_{4}=450$.

The figures below show the reinsurer's part of each claim (light grey column) and the provided cover before (black column) and after the occurred claims (dark grey column). 
Additionally the mottled and white columns refer to the amount used from the first and second reinstatement, respectively. The premiums are paid pro-rata-capita:

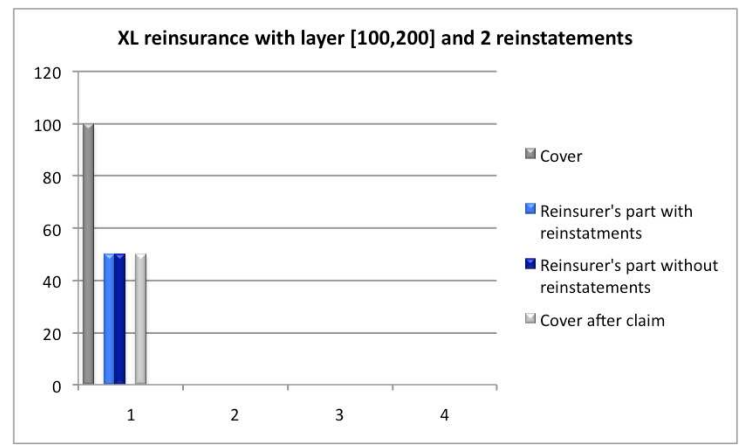

(a) First claim - reduction of available cover

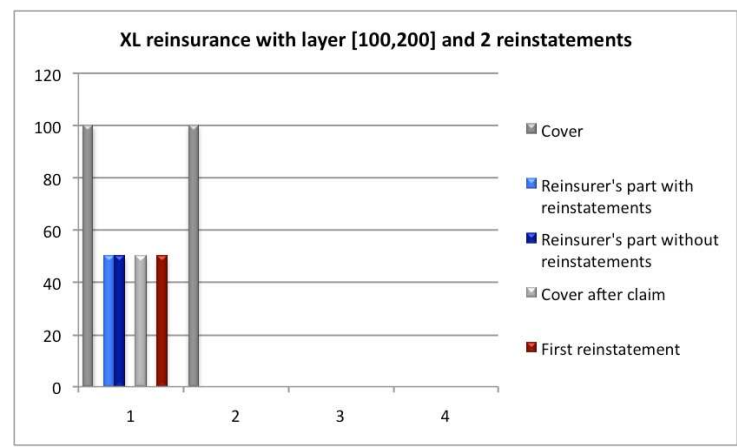

(b) First reinstatement - full cover

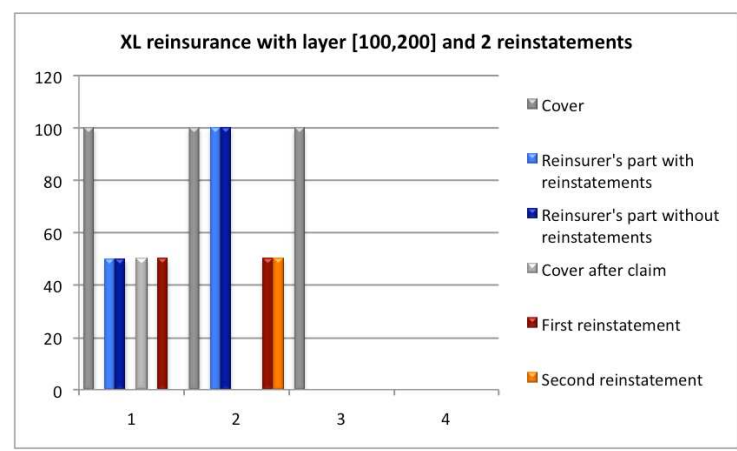

(d) First and second reinstatement - full cover

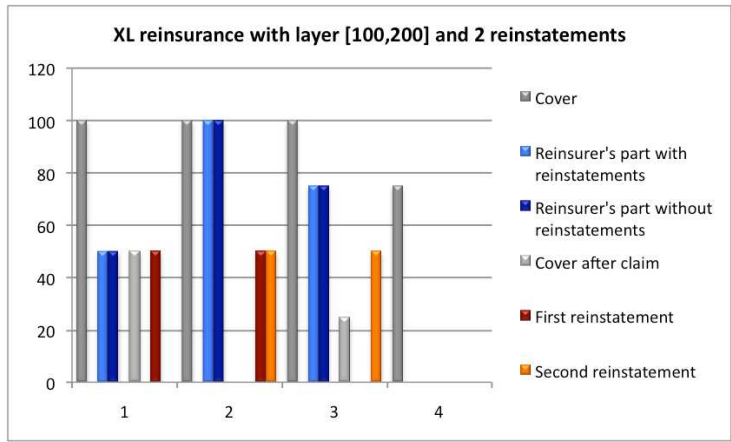

(f) Second reinstatement - no full cover

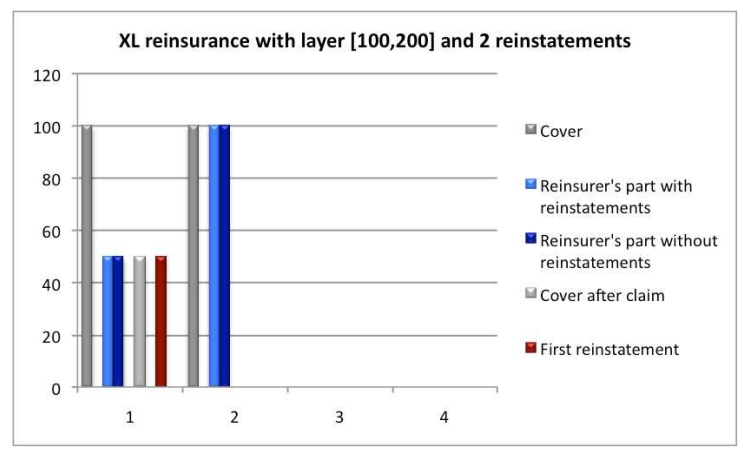

(c) Second claim - no cover left

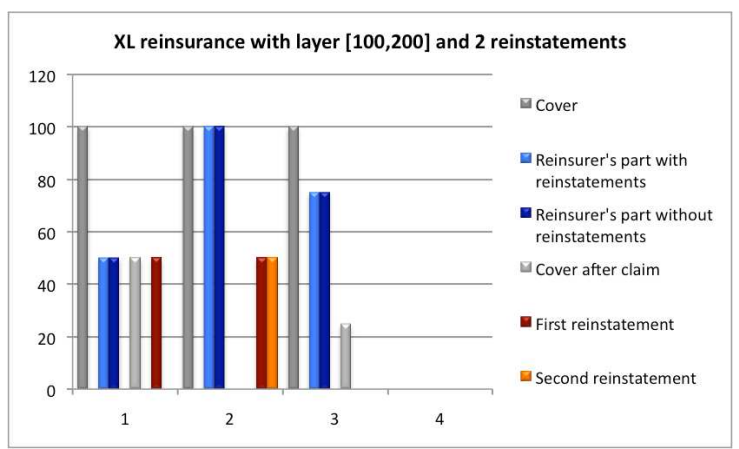

(e) Third claim - reduction of available cover

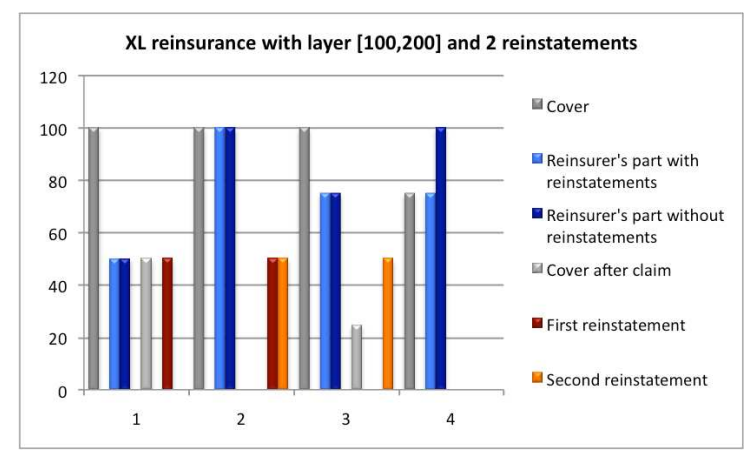

(g) Fourth claim - no cover left

Figure 1: Example of an XL reinsurance with two reinstatements 
At the beginning of the contract the cedent pays an initial premium $p_{0}$ for the first cover (see Figure 1(a), black column). After the first claim the cover is reduced to 50 (dark grey column) and therefore the cedent refills the cover again to 100 by using 50 of the first reinstatement (see Figure 1(b), mottled column). For this reinstatement the cedent has to pay $\frac{50}{100} 0.5 p_{0}=0.25 p_{0}$. The second claim used up the cover completely and hence the cedent reinstates the cover with 50 from the first reinstatement (mottled column) and 50 of the second one (white column), see Figures 1(c) and 1(d). The cedent's payment is $\frac{50}{100} 0.5 p_{0}$ for the first and $\frac{50}{100} 0.5 p_{0}$ for the second reinstatement.

In Figure 1(g) we see that after the third claim the cover was reinstated to 75 , since only 50 of the second reinstatement were left. The reinsurer's part of the last claim is therefore 75 , the cover and the two reinstatements are used up completely, so the contract closes.

The total premium income of the reinsurer is therefore $2 p_{0}$.

The surplus process of the cedent under such a reinsurance contract is given by

$$
R_{X L}(t)=u_{0}-p_{0}+\beta t-\sum_{j=1}^{N_{t}} C_{j}-P_{X L}(t),
$$

where $P_{X L}(t)$ is the amount of reinstatement premiums paid up to time $t$. The total amount of charged reinstatement premiums at the end of the period of cover is therefore $p_{0}\left(1+\frac{c}{m} \sum_{j=1}^{k} \min (\max (Z-(j-1) m, 0), m)\right)$.

The resulting ruin probability is denoted by $\psi_{X L}(u, T)$. In the following, the initial surplus $u_{0}$ is assumed to be already reduced by the initial premium $p_{0}$, so we define $u:=u_{0}-p_{0}$.

\section{A reinstatement model with PDMP's}

Since our focus is on the determination of the finite-time ruin probability of the cedent under an XL reinsurance contract with reinstatement clauses, it is useful to markovize this a priori non-Markovian process. This can be achieved by extending the model description as a two-dimensional process, with the current amount of used reinsurance cover as a second dimension. We use piecewise deterministic Markov processes to describe the resulting process.

The state space of the process $X(t)$ is denoted by $E=\mathbb{R} \times[0,(k+1) m]$, where the first component, say $u_{t}$, denotes the surplus of the cedent at time $t$ and the second component, say $r_{t}$, the reinsurance cover used up to time $t$. The construction of the PDMP can be split up into the deterministic part and the jumps of the process. The dynamics of the process between jumps can be described by the integral curve $\phi((u, r), t)=(u+\beta t, r)$. Thus the deterministic part of the Markov process is determined by

$$
\phi\left(\left(u_{T_{k-1}}, r_{T_{k-1}}\right), t\right)=\left(u_{T_{k-1}}+\beta\left(t-T_{k-1}\right), r_{T_{k-1}}\right),
$$

for $T_{k-1} \leq t<T_{k}$, where $T_{k}$ is the arrival time of the $k$-th claim. (Clearly the reinsurance cover remains constant between jumps.) 
Define

$$
\begin{aligned}
\text { layer } & =\min (\max (x-l, 0), m), \\
l(x, r)_{k} & =\min (\text { layer },(k+1) m-r)
\end{aligned}
$$

and $x^{*}(u, r, t)$ as the solution (w.r.t. $\left.x\right)$ of

$$
u+\beta t-x+l(x, r)_{k}-\frac{c p_{0}}{m} l(x, r)_{k-1}=0,
$$

where $x$ denotes the occurred claim size and $r$ the reinsurance cover already used up.

From the general form of the generator of a PDMP as given in Theorem 5.5 in Davis [3], the generator of the PDMP describing the surplus process $R_{X L}(t)$ of the cedent under XL reinsurance with reinstatements is given by

$$
\begin{aligned}
\mathscr{A} g((u, r), t) & =\frac{\partial}{\partial t} g((u, r), t)+\beta \frac{\partial}{\partial u} g((u, r), t) \\
& +\lambda \int_{0}^{x^{*}(u, r, t)} g\left(\left(u+\beta t-x+l(x, r)_{k}-\frac{c p_{0}}{m} l(x, r)_{k-1}, r+l(x, r)_{k}\right), t\right) d F(x) \\
& -\lambda g((u, r), t)+\lambda \int_{x^{*}(u, r, t)}^{\infty} d F(x),
\end{aligned}
$$

where $F(x)$ denotes the claim size distribution of the claims $X_{i}$ of the process and $g$ is some function in the domain $\mathcal{D}(\mathscr{A})$ of $\mathscr{A}$.

Theorem 3.1: Let $T$ be fixed. If $g((u, r), t) \in \mathcal{D}(\mathscr{A})$ is a solution of $\mathscr{A} g=0$ on $(\mathbb{R} \times$ $[0,(k+1) m]) \times[0, T]$ with boundary condition $g((u, r), T)=\mathbf{1}_{u<0}$, then

$$
\psi_{X L}(u, T)=\mathbb{P}(\tau(u)<T)=g((u, r), 0) .
$$

Proof: In analogy to the proof of Theorem 11.3.3 in Rolski et al. [9], where it is shown that the survival probability can be expressed via PDMP's, we use results from martingale theory. We know that $\{g(X(t), t), t \geq 0\}$ is a martingale, and so is $\{g(X(\tau(u) \wedge t), t), t \geq 0\}$ and consequently also $\{g(X(\tau \wedge t \wedge T), t \wedge T) t \geq 0\}$. Therefore we get

$$
g((u, r), 0)=\mathbb{E}[g(X(t), t)]=\mathbb{E}[g(X(\tau(u) \wedge T), T)]=\mathbb{P}(\tau(u)<T),
$$

since $g(X(\tau(u)), T)=0$ whenever $\tau(u) \geq T$.

Note that $\psi_{X L}(u, T)$ is indeed in the domain of the generator $\mathscr{A}$, since the respective conditions of Theorem 5.5 in Davis [3] are fulfilled (for a more detailed discussion of this point see also Section 11.3.4 in Rolski et al. [9]).

Altogether, one first has to solve the partial integro-differential equation $\mathscr{A} g=0$ for $g((u, r), t)$ with $(2)$ and then $g((u, r), 0)$ is the required expression for the finite-time ruin probability $\psi_{X L}(u, T)$.

An analytic solution of this problem is in general not possible. One can instead numerically solve the partial integro-differential equation $\mathscr{A} g=0$, but we shall follow another approach in the following section, which leads to a more effective numerical technique. 


\section{An integral equation for the ruin probability}

Alternatively to the approach of the previous section, one can condition on the time and size of the first claim to obtain the following integral operator (here $x^{*}(u, r, t)$ denotes the solution w.r.t. $x$ of equation (1)):

$$
\begin{aligned}
\mathcal{A} g((u, r), T)= & \int_{0}^{T} \lambda \exp (-\lambda t) \int_{0}^{x^{*}(u, r, t)} g\left(\left(u+\beta t-x+l(x, r)_{k}-\frac{c p_{0}}{m} l(x, r)_{k-1},\right.\right. \\
& \left.\left.r+l(x, r)_{k}\right), T-t\right) d F(x) d t+\int_{0}^{T} \lambda \exp (-\lambda t) \int_{x^{*}(u, r, t)}^{\infty} d F(x) d t .
\end{aligned}
$$

Obviously $\psi_{X L}(u, T)$ is a fixed point of this integral operator. Furthermore, it is shown in the appendix , that the solution of (2) and the fixed point of (3) coincide. $\mathcal{A}$ is a contraction in the Banach space of all bounded functions, because

$$
\begin{aligned}
& \left\|\mathcal{A} g_{1}((u, r), t)-\mathcal{A} g_{2}((u, r), t)\right\|_{\infty}= \\
= & \left\|\int_{0}^{T} \lambda \exp (-\lambda t) \int_{0}^{x^{*}(u, r, t)}\left(g_{1}(k((u, r), t))-g_{2}(k((u, r), t))\right) d F(x) d t\right\|_{\infty} \\
\leq & \left\|g_{1}-g_{2}\right\|_{\infty} \int_{0}^{T} \lambda \exp (-\lambda t) d t=\left\|g_{1}-g_{2}\right\|_{\infty} \cdot(1-\exp (-\lambda T)),
\end{aligned}
$$

where $k((u, r), t)=\left(\left(u+\beta t-x+l(x, r)_{k}-\frac{c p_{0}}{m} l(x, r)_{k-1}, r+l(x, r)_{k}\right), T-t\right)$.

For computational purposes the fixed point approach is more suitable. By iterating the operator $\mathcal{A} d$ times on some starting function $g^{(0)}((u, r), t)$, i.e.

$$
g^{(d)}((u, r), t)=\mathcal{A}^{d} g^{(0)}((u, r), t)
$$

and evaluating the resulting $2 d$-dimensional integral by Monte Carlo techniques, one obtains an approximation for $\psi_{X L}(u, T)$. This approach is further discussed in Section 5 . The appropriate choice of the starting function is discussed in Section 6 .

\section{A recursive algorithm for the numerical solution}

In the following, we consider exponentially distributed claim sizes (parameter $\gamma$ ). Then the operator $\mathcal{A}$ can be written as

$$
\begin{aligned}
\mathcal{A} g((u, r), T)= & \int_{0}^{T} \lambda \exp (-\lambda t) \int_{0}^{x^{*}(u, r, t)} g\left(\left(u+\beta t-x+l(x, r)_{k}-\frac{c p_{0}}{m} l(x, r)_{k-1}\right.\right. \\
& \left.\left.r+l(x, r)_{k}\right), T-t\right) \gamma \exp (-\gamma x) d x d t \\
+ & \int_{0}^{T} \lambda \exp (-\lambda t) \int_{x^{*}(u, r, t)}^{\infty} \gamma \exp (-\gamma x) d x d t .
\end{aligned}
$$


To apply Monte Carlo methods in an effective way, we first transform the integration domain of the operator to the unit cube by applying the techniques of Albrecher et al. [1] to the present setup. This results in

$$
\begin{aligned}
\mathcal{A} g((u, r), T)= & (1-\exp (-\lambda T)) \int_{0}^{1} \int_{0}^{1}\left(1-\exp \left(-\gamma x^{*}(u, r, t)\right)\right) \\
& g\left(\left(u+\beta t-x+l(x, r)_{k}-\frac{c p_{0}}{m} l(x, r)_{k-1}, r+l(x, r)_{k}\right), T-t\right) d v d w \\
+ & (1-\exp (-\lambda T)) \int_{0}^{1} \int_{0}^{1} \exp \left(-\gamma x^{*}(u, r, t)\right) d v d w .
\end{aligned}
$$

Here $t$ and $x$ are defined by

$$
\begin{aligned}
t & =-\frac{\log (1-w(1-\exp (-\lambda T)))}{\lambda} \\
x & =-\frac{\log \left(1-v\left(1-\exp \left(-\gamma x^{*}(u, r, t)\right)\right)\right)}{\gamma}
\end{aligned}
$$

and $x^{*}(u, r, t)$ is the solution of equation (1) and can be calculated in every iteration step. The integral operator is now applied $d$ times onto $g^{(0)}((u, r), t)$ and the resulting multidimensional integral $g^{(d)}((u, r), t)$ is approximated by the Monte Carlo estimate

$$
g^{(d)}((u, r), t) \approx \frac{1}{N} \sum_{n=1}^{N} g_{n}^{(d)}((u, r), t),
$$

where each $g_{n}^{(d)}((u, r), t)$ is based on a uniformly distributed point $\mathbf{x}_{n} \in[0,1]^{2 d}$ (note that $d$ will usually be quite large) and calculated by the recursion

$$
\begin{aligned}
g_{n}^{(0)}((u, r), t) & =g^{(0)}((u, r), t) \\
g_{n}^{(i)}((u, r), t)= & (1-\exp (-\lambda t))\left(1-\exp \left(-\gamma x^{*}\left(u, r, t_{n}^{i}\right)\right)\right) \\
& \cdot g_{n}^{(i-1)}\left(\left(u+\beta t_{n}^{i}-x_{n}^{i}+l\left(x_{n}^{i}, r\right)_{k}-\frac{c p_{0}}{m} l\left(x_{n}^{i}, r\right)_{k-1}, r+l\left(x_{n}^{i}, r\right)_{k}\right), t-t_{n}^{i}\right) \\
+ & (1-\exp (-\lambda t)) \exp \left(-\gamma x^{*}\left(u, r, t_{n}^{i}\right)\right) .
\end{aligned}
$$

Here $t_{n}^{i}$ and $x_{n}^{i}$ are determined according to equation (4) for two components $v$ and $w$ of the unit interval $[0,1]^{2 d}$.

The recursive algorithm in formula (6) is implemented in Mathematica. It is essential that $\frac{c p_{0}}{m} l\left(x_{n}^{i}, y\right)_{k-1}$ is always larger or equal to 0 , in order to avoid negative premiums. Therefore in the implementation $\frac{c p_{0}}{m} l\left(x_{n}^{i}, y\right)_{k-1}$ is replaced by $\frac{c p_{0}}{m} \max \left(l\left(x_{n}^{i}, y\right)_{k-1}, 0\right)$.

\section{$6 \quad$ Numerical illustrations}

We now illustrate the performance of the resulting algorithm and analyze the effect of XL reinsurance with reinstatements on the ruin probability of the cedent.

Throughout this section we fix the parameter values as summarized in Table 1. 


\begin{tabular}{|lc|}
\hline Parameter of the Poisson process & $\lambda=10$ \\
Parameter of the exponentially distributed claim sizes & $\gamma=\frac{1}{5}$ \\
Covered layer within each reinstatement & {$[l, l+m]=[6,6+15]$} \\
Duration of the reinsurance contract & $T=1$ \\
Simulation runs for MC estimates & $N=75000$ \\
Level of confidence interval & $95 \%$ \\
\hline
\end{tabular}

Table 1: Parameter values

Furthermore we assume that both the cedent's premium and the reinsurance premium are determined by the expected value principle.

The random numbers are generated using the algorithm ExtendedCA in Mathematica 6.0, which uses cellular automata based on a particular five-neighbor rule to generate high-quality pseudo-random numbers.

\subsection{Accuracy of results under various starting functions}

In a first step we evaluate the influence of the recursion depth $d$ and the starting function $g^{(0)}((u, r), t)$ on the accuracy of the results.

Fix the security loading of the cedent with $\alpha_{c e d}=0.2$ and of the reinsurer with $\alpha_{r e}=0.3$. Additionally, the initial surplus of the cedent is assumed to be $u=40$ and for the recursion depth we use three different choices $(d=20, d=35$ and $d=70)$ to assess the effect of the recursion depth on the performance. Consequently we generate up to 140-dimensional random numbers $\mathbf{x}_{n}$.

We first choose the trivial starting function $g^{(0)}=g^{(0)}((u, r), t)=0$, if $u \geq 0$. For $u<0$ we set $g^{(0)}=g^{(0)}((u, r), t)=1$. The following table shows the effect of excess of loss reinsurance (without reinstatements, but an upper coverage limit of 15) on the probability of ruin for $d=70$.

\begin{tabular}{|l|l|}
\hline \multicolumn{2}{|c|}{ Ruin Probability without reinsurance } \\
\hline$\psi(40,1)$ using formula $(7):$ \\
\hline \multicolumn{2}{|c|}{ Ruin Probability with reinsurance } \\
\hline$\psi_{X L}(40,1): \quad 0.045820$ & Confidence interval: $[0.044790,0.046851]$ \\
\hline
\end{tabular}

Table 2: Effects of the excess of loss reinsurance on the probability of ruin

So in this case the classical excess of loss reinsurance layer $[6,21]$ (i.e. no additional reinstatements are available after the consumption of the reinsurance cover) improves the probability of ruin by $13 \%$.

Tables 3 and 4 summarize the probabilities of ruin of the cedent and the corresponding confidence intervals for different combinations of offered reinstatements $k$ and charged premium percentages $c$. Here one sees that $\psi_{X L}(40,1)$ is monotonically increasing in $c$, but that it is not monotone in $k$, i.e. an increase of the number of reinstatements does not 
automatically decrease $\psi(u)$. This comes from the balance between reduced risk due to higher reinsurance cover, but at the same time higher costs for the reinsurance premiums.

\begin{tabular}{|ll|ll|}
\hline$\psi_{X L}(40,1)$ with $c=0 .:$ & 0.024310 & Confidence interval: & {$[0.023542,0.025078]$} \\
$\psi_{X L}(40,1)$ with $c=0.5:$ & 0.029833 & Confidence interval: & {$[0.028979,0.030686]$} \\
$\psi_{X L}(40,1)$ with $c=1 .:$ & 0.033785 & Confidence interval: & {$[0.032877,0.034694]$} \\
$\psi_{X L}(40,1)$ with $c=1.5:$ & 0.036809 & Confidence interval: & {$[0.035860,0.037758]$} \\
\hline
\end{tabular}

Table 3: Effects of $k=1$ reinstatements on the probability of ruin

\begin{tabular}{|ll|lll}
\hline$\psi_{X L}(40,1)$ with $c=0 .:$ & 0.014954 & Confidence interval: & {$[0.014319,0.015589]$} \\
$\psi_{X L}(40,1)$ with $c=0.5:$ & 0.032902 & Confidence interval: & {$[0.031982,0.033821]$} \\
$\psi_{X L}(40,1)$ with $c=1 .:$ & 0.046573 & Confidence interval: & {$[0.045498,0.047648]$} \\
$\psi_{X L}(40,1)$ with $c=1.5:$ & 0.055864 & Confidence interval: & {$[0.054701,0.057027]$} \\
\hline
\end{tabular}

Table 4: Effects of $k=3$ reinstatements on the probability of ruin

In a straight-forward implementation of the recursion algorithm, each value given above needs approximately 1 hour computation time on a standard PC (this can certainly be improved by a more efficient vector implementation, but note also that the reinstatement clauses entail a lot of algebraic operations in each integration run). It is natural to ask whether a reduction of the recursion depth $d$ (which will decrease the computation time proportionally) can be afforded in this method in terms of accuracy. Table 5 shows indeed that for $d=35$ the obtained results are very similar to those calculated with recursion depth $d=70$ (and the computation time is approximately halved). One observes that for an increasing number of reinstatements $k$ and fixed $c$, the difference between the results using $d=70$ and $d=35$ becomes smaller.

\begin{tabular}{|l||c|c||c|c|}
\hline & $k=1$ & confidence interval & $k=3$ & confidence interval \\
\hline$c=0$. & 0.023953 & {$[0.023192,0.024715]$} & 0.014829 & {$[0.014203,0.015455]$} \\
\hline$c=0.5$ & 0.029335 & {$[0.028491,0.030179]$} & 0.032423 & {$[0.031506,0.033340]$} \\
\hline$c=1$ & 0.033265 & {$[0.032365,0.034165]$} & 0.045881 & {$[0.044811,0.046951]$} \\
\hline$c=1.5$ & 0.036260 & {$[0.035320,0.037200]$} & 0.055094 & {$[0.053936,0.056252]$} \\
\hline
\end{tabular}

Table 5: $\psi_{X L}(40,1)$ using a recursion depth of $d=35$

\begin{tabular}{|l||c|c||c|c|}
\hline & $k=1$ & confidence interval & $k=3$ & confidence interval \\
\hline$c=0$. & 0.023648 & {$[0.022898,0.024397]$} & 0.014474 & {$[0.013848,0.015099]$} \\
\hline$c=0.5$ & 0.029160 & {$[0.028323,0.029997]$} & 0.032555 & {$[0.031638,0.033472]$} \\
\hline$c=1$ & 0.033178 & {$[0.032283,0.034073]$} & 0.046310 & {$[0.045235,0.047384]$} \\
\hline$c=1.5$ & 0.036142 & {$[0.035206,0.037078]$} & 0.055580 & {$[0.054418,0.056743]$} \\
\hline
\end{tabular}

Table 6: $\psi_{X L}(40,1)$ using a recursion depth of $d=20$ 
As Table 6 shows, a further decrease of recursion depth to $d=20$ still leads to comparable results, but the third digit now is not accurate and it will depend on the concrete application whether this reduced accuracy is still acceptable.

In absence of analytical solutions, we compare these results with simulated ruin probabilities using stochastic simulation of surplus paths of the underlying risk process according to the compound Poisson dynamics. Table 7 depicts the ruin probabilities for several combinations of $k$ and $c$ (in each case, the estimate is based on 500,000 simulation runs).

\begin{tabular}{|l||c|c||c|c|}
\hline & $k=1$ & confidence interval & $k=3$ & confidence interval \\
\hline$c=0$. & 0.024016 & {$[0.023587,0.024446]$} & 0.015116 & {$[0.014775,0.015457]$} \\
\hline$c=0.5$ & 0.029784 & {$[0.029306,0.030262]$} & 0.032588 & {$[0.032088,0.033088]$} \\
\hline$c=1$ & 0.033296 & {$[0.032790,0.033802]$} & 0.045988 & {$[0.045394,0.046582]$} \\
\hline$c=1.5$ & 0.036634 & {$[0.036104,0.037165]$} & 0.055636 & {$[0.054982,0.056290]$} \\
\hline
\end{tabular}

Table 7: $\psi_{X L}(40,1)$ using the simulation of the risk process

The ruin probabilities obtained by the recursive numerical method above nicely match the values obtained by simulation (as long as $d$ is chosen sufficiently high).

In the following we investigate whether a more sophisticated starting function $g^{(0)}((u, r), t)$ leads to significantly improved results. A natural choice for a better starting function are the infinite-time and finite-time ruin probabilities without reinsurance, $\psi(u)$ and $\psi(u, t)$. Since the claim size distribution is exponential (parameter $\gamma$ ), there exist closed-form expressions for the latter, namely $\psi(u)=\frac{\lambda}{\gamma \beta} \exp \left(-u\left(\gamma-\frac{\lambda}{\beta}\right)\right)$ and

$$
\psi(u, t)=1-\exp (-\gamma u-(1+\tau) \lambda t) \omega(\gamma u+\tau \lambda t, \lambda t)
$$

where $\tau=\gamma \beta / \lambda$ and

$$
\begin{aligned}
\omega(z, \theta)=J(\theta z)+\theta J^{(1)}(\theta z) & +\int_{0}^{z} \exp (z-v) J(\theta v) d v \\
& -\frac{1}{\tau} \int_{0}^{\tau \theta} \exp (\tau \theta-v) J\left(z \tau^{-1} v\right) d v
\end{aligned}
$$

Here $J(x)=I_{0}(2 \sqrt{x})$ and $I_{0}(x)$ denotes the modified Bessel function (see e.g. Rolski et al. [9]).

Table 8 gives the resulting ruin probabilities for $c=100 \%$ and recursion depths $d=35$ and $d=20$. 


\begin{tabular}{|l||c|c||c|c|}
\hline \multicolumn{1}{|l||}{$d=35$} & \multicolumn{2}{c||}{$g^{(0)}((u, r), t)=\psi(u)$} & \multicolumn{2}{c|}{$g^{(0)}((u, r), t)=\psi(u, t)$} \\
\hline & probability & confidence interval & probability & confidence interval \\
\hline$k=1$ & 0.033501 & {$[0.032595,0.034407]$} & 0.033382 & {$[0.032487,0.034277]$} \\
\hline$k=3$ & 0.046526 & {$[0.045451,0.047601]$} & 0.046144 & {$[0.045074,0.047214]$} \\
\hline \hline$d=20$ & \multicolumn{2}{|c|}{$g^{(0)}((u, r), t)=\psi(u)$} & \multicolumn{2}{|c|}{$g^{(0)}((u, r), t)=\psi(u, t)$} \\
\hline & probability & confidence interval & probability & confidence interval \\
\hline$k=1$ & 0.034601 & {$[0.033696,0.035506]$} & 0.033683 & {$[0.032777,0.034590]$} \\
\hline$k=3$ & 0.047735 & {$[0.046652,0.048818]$} & 0.046294 & {$[0.045224,0.047363]$} \\
\hline
\end{tabular}

Table 8: $\psi_{X L}(40,1)$ using different starting functions $g^{(0)}$

One observes that for the starting function $g^{(0)}((u, r), t)=\psi(u)$ a recursion depth of $d=20$ leads to slightly over-estimated values for the ruin probability. Increasing $d$ and using the finite-time ruin probability $\psi(u, t)$ as the starting function significantly improves these results. Note that the resulting ruin probabilities for this starting function and $d=20$ are closer to the results of the simulation of the risk process than the ruin probabilities calculated with the simple starting function and $d=70$.

\subsection{Analysis of different levels of initial surplus $u$}

In this section we analyze the effect of XL reinsurance with reinstatements for various levels of initial surplus $u$. We keep the security loadings of cedent and reinsurer fixed with $\alpha_{\text {ced }}=0.2$ and $\alpha_{r e}=0.3$ and study the ruin probability of the cedent with $k=1$ and $k=3$ reinstatements. The recursion depth is set to $d=20$ and to $d=35$ to evaluate the stability of the results. As starting function we use $\psi(u, t)$.

The following tables give the ruin probabilities for $u \in\{20,40,60,80\}$ (here $c=100 \%$ ). Table 9 shows that an increasing number of reinstatements reduces the probability of ruin. At the same time it is preferable not to have any reinsurance, because the premium payment close to ruin is more risky than covering the potential claims.

\begin{tabular}{|l||c|c|}
\hline$d=20$ & $\psi_{X L}(20,1)$ & confidence interval \\
\hline$k=1$ & 0.314771 & {$[0.312447,0.317094]$} \\
$k=3$ & 0.300447 & {$[0.298144,0.302749]$} \\
\hline \hline$d=35$ & $\psi_{X L}(20,1)$ & confidence interval \\
\hline$k=1$ & 0.312863 & {$[0.310549,0.315178]$} \\
$k=3$ & 0.298747 & {$[0.296454,0.301041]$} \\
\hline \hline
\end{tabular}

Without reinsurance: $\psi(20,1)=0.221820$

Table 9: The probability of ruin with initial surplus $u=20$

With increasing initial surplus $u$ the situation changes and the cedent can reduce the ruin probability by agreeing to a reinsurance. The following tables show that on from some 
threshold level $u$, the ruin probability is always decreased by reinsurance irrespectively of the number of reinstatements. One can observe from the tables that for medium sizes of $u$ a larger number of reinstatements does not automatically lead to more safety. If $u$ is high enough, the ruin probability again decreases for an increasing number of reinstatements (see Figure 2).

\begin{tabular}{|l||c|c|}
\hline$d=20$ & $\psi_{X L}(40,1)$ & confidence interval \\
\hline$k=1$ & 0.033683 & {$[0.032777,0.034590]$} \\
$k=3$ & 0.046294 & {$[0.045224,0.047363]$} \\
\hline \hline$d=35$ & $\psi_{X L}(40,1)$ & confidence interval \\
\hline$k=1$ & 0.033382 & {$[0.032487,0.034277]$} \\
$k=3$ & 0.046144 & {$[0.045074,0.047214]$} \\
\hline \hline \multicolumn{3}{|l|}{ Without reinsurance: $\psi(40,1)=0.052907$} \\
\hline
\end{tabular}

Table 10: The probability of ruin with initial surplus $u=40$

\begin{tabular}{|l|c|c|}
\hline$d=20$ & $\psi_{X L}(60,1)$ & confidence interval \\
\hline$k=1$ & 0.005115 & {$[0.004784,0.005446]$} \\
$k=3$ & 0.004907 & {$[0.004568,0.005245]$} \\
\hline \hline$d=35$ & $\psi_{X L}(60,1)$ & confidence interval \\
\hline$k=1$ & 0.004777 & {$[0.004466,0.005089]$} \\
$k=3$ & 0.004779 & {$[0.004452,0.005106]$} \\
\hline \hline \multicolumn{3}{|l}{ Without reinsurance $\psi(60,1)=0.010523$} \\
\hline
\end{tabular}

Table 11: The probability of ruin with initial surplus $u=60$

\begin{tabular}{|l||c|c|}
\hline$d=20$ & $\psi_{X L}(80,1)$ & confidence interval \\
\hline$k=1$ & 0.000791 & {$[0.000667,0.000915]$} \\
$k=3$ & 0.000390 & {$[0.000306,0.000475]$} \\
\hline \hline$d=35$ & $\psi_{X L}(80,1)$ & confidence interval \\
\hline$k=1$ & 0.000742 & {$[0.000628,0.000855]$} \\
$k=3$ & 0.000345 & {$[0.000268,0.000422]$} \\
\hline \hline \multicolumn{3}{|l|}{ Without reinsurance $\psi(80,1)=0.001799$} \\
\hline
\end{tabular}

Table 12: The probability of ruin with initial surplus $u=80$

Table 13 shows that the numerical results are in agreement with the simulation results (where each estimate is based on 500,000 simulation runs). 


\begin{tabular}{|c||c|c||c|c|}
\hline & $k=1$ & confidence interval & $k=3$ & confidence interval \\
\hline$u=20$ & 0.313524 & {$[0.311972,0.315076]$} & 0.299342 & {$[0.297825,0.300859]$} \\
\hline$u=40$ & 0.033296 & {$[0.032790,0.033802]$} & 0.045988 & {$[0.045394,0.046582]$} \\
\hline$u=60$ & 0.004934 & {$[0.004739,0.005129]$} & 0.004962 & {$[0.004767,0.005157]$} \\
\hline$u=80$ & 0.000746 & {$[0.000670,0.000822]$} & 0.000364 & {$[0.000311,0.000417]$} \\
\hline
\end{tabular}

Table 13: $\psi_{X L}(u, 1)$ using simulation of the risk process

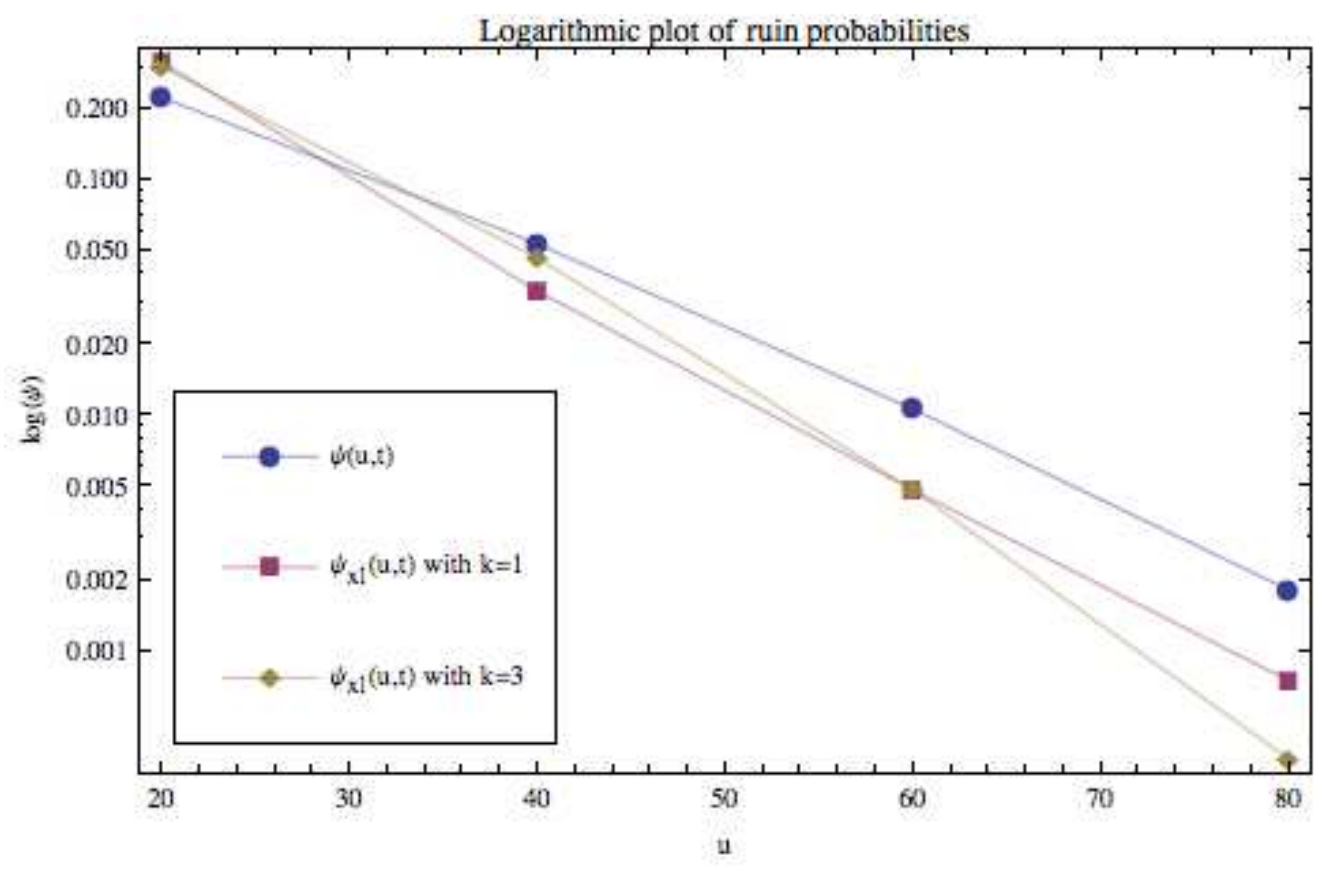

Figure 2: Logarithmic plot of ruin probabilities w.r.t. $u$, for $k \in\{1,3\}$

\subsection{Analysis of different combinations of security loadings}

Let us finally consider the effect of the security loading on the ruin probability of the cedent (we fix $u=40$ and $d=35$ ). As in the previous section we use the finite-time ruin probability $\psi(u, t)$ as starting function for the algorithm and evaluate the ruin probability of the cedent under XL reinsurance with $k=1$ and $k=3$ reinstatements. The charged premium for each reinstatement is assumed to be $100 \%$ of the initial premium $p_{0}$.

For the security loading of the cedent we analyze the levels $\alpha_{\text {ced }}=0.2$ and $\alpha_{\text {ced }}=0.3$ and combine these values with different security loadings of the reinsurer $\alpha_{r e}$, where we assume as restriction that $\alpha_{c e d}<\alpha_{r e}$.

For $\alpha_{\text {ced }}=0.2$ we investigate the effects of reinstatements, if the reinsurer charges a security loading of $\alpha_{r e}=0.3, \alpha_{r e}=0.4$ and $\alpha_{r e}=0.5$, respectively. 


\begin{tabular}{|l||c|c|}
\hline$\alpha_{r e}=0.3$ & $\psi_{X L}(40,1)$ & confidence interval \\
\hline$k=1$ & 0.033382 & {$[0.032487,0.034277]$} \\
$k=3$ & 0.046144 & {$[0.045074,0.047214]$} \\
\hline \hline$\alpha_{r e}=0.4$ & $\psi_{X L}(40,1)$ & confidence interval \\
\hline$k=1$ & 0.040185 & {$[0.039192,0.041178]$} \\
$k=3$ & 0.057548 & {$[0.056361,0.058735]$} \\
\hline \hline$\alpha_{r e}=0.5$ & $\psi_{X L}(40,1)$ & confidence interval \\
\hline$k=1$ & 0.047978 & {$[0.046891,0.049066]$} \\
$k=3$ & 0.069778 & {$[0.068482,0.071075]$} \\
\hline \hline \multicolumn{3}{|c|}{ Without reinsurance: $\psi(40,1)=0.052907$} \\
\hline
\end{tabular}

Table 14: The probability of ruin with $\alpha_{c e d}=0.2$

Table 14 summarizes the ruin probabilities and the confidence intervals for $\alpha_{c e d}=0.2$ : First one notes that here reinsurance with $k=1$ reinstatements improves the cedent's situation in each case (this was not always the case for small values of $u$ in Table 9 in Section 6.2) and again an increase in the number of reinstatements can make the cedent's situation more risky than without reinsurance.

Hence depending on $u$ and the difference between the cedent's and the reinsurer's security loading, it may be preferable to have no reinsurance. Table 15 shows a similar behavior of the ruin probability for increasing security loadings $\alpha_{r e}$ (with $\alpha_{r e} \in\{0.35,0.4,0.5\}$ ), if the cedent charges $\alpha_{\text {ced }}=0.3$.

\begin{tabular}{|l||c|c|}
\hline$\alpha_{r e}=0.35$ & $\psi_{X L}(40,1)$ & confidence interval \\
\hline$k=1$ & 0.027308 & {$[0.026506,0.028109]$} \\
$k=3$ & 0.038735 & {$[0.037769,0.039701]$} \\
\hline \hline$\alpha_{r e}=0.4$ & $\psi_{X L}(40,1)$ & confidence interval \\
\hline$k=1$ & 0.030061 & {$[0.029222,0.030900]$} \\
$k=3$ & 0.043536 & {$[0.042513,0.044559]$} \\
\hline \hline$\alpha_{r e}=0.5$ & $\psi_{X L}(40,1)$ & confidence interval \\
\hline$k=1$ & 0.035367 & {$[0.034455,0.036278]$} \\
$k=3$ & 0.052425 & {$[0.051315,0.053535]$} \\
\hline \hline \multicolumn{3}{|l|}{ Without reinsurance: $\psi(40,1)=0.041291$} \\
\hline
\end{tabular}

Table 15: The probability of ruin with $\alpha_{c e d}=0.3$

In addition one notices that the higher security loading of the cedent obviously leads to a decreased ruin probability in the case without reinsurance. Therefore the difference between the security loadings $\alpha_{c e d}=0.3$ and $\alpha_{r e}$ has to be smaller than in the case with $\alpha_{c e d}=0.2$ to achieve an improvement for the cedent's situation for $k>1$ reinstatements. We now compare these results with the estimated ruin probabilities evaluated by simulating the cedent's surplus process, see Table 16 and Table 17. 


\begin{tabular}{|l||c|c|}
\hline$\alpha_{r e}=0.3$ & $\psi_{X L}(40,1)$ & confidence interval \\
\hline$k=1$ & 0.033296 & {$[0.032790,0.033802]$} \\
$k=3$ & 0.045988 & {$[0.045394,0.046582]$} \\
\hline \hline$\alpha_{r e}=0.4$ & $\psi_{X L}(40,1)$ & confidence interval \\
\hline$k=1$ & 0.040398 & {$[0.039841,0.040955]$} \\
$k=3$ & 0.057084 & {$[0.056422,0.057746]$} \\
\hline \hline$\alpha_{r e}=0.5$ & $\psi_{X L}(40,1)$ & confidence interval \\
\hline$k=1$ & 0.047704 & {$[0.047099,0.048309]$} \\
$k=3$ & 0.069266 & {$[0.068537,0.069996]$} \\
\hline
\end{tabular}

Table 16: The probability of ruin using simulation of the risk process with $\alpha_{c e d}=0.2$

\begin{tabular}{|l||c|c|}
\hline$\alpha_{r e}=0.35$ & $\psi_{X L}(40,1)$ & confidence interval \\
\hline$k=1$ & 0.027418 & {$[0.026959,0.027877]$} \\
$k=3$ & 0.038832 & {$[0.038286,0.039378]$} \\
\hline \hline$\alpha_{r e}=0.4$ & $\psi_{X L}(40,1)$ & confidence interval \\
\hline$k=1$ & 0.030022 & {$[0.029542,0.030502]$} \\
$k=3$ & 0.042984 & {$[0.042409,0.043559]$} \\
\hline \hline$\alpha_{r e}=0.5$ & $\psi_{X L}(40,1)$ & confidence interval \\
\hline$k=1$ & 0.035310 & {$[0.034789,0.035831]$} \\
$k=3$ & 0.052630 & {$[0.051994,0.053266]$} \\
\hline
\end{tabular}

Table 17: The probability of ruin using simulation of the risk process with $\alpha_{c e d}=0.3$

Altogether, XL reinsurance with reinstatements improves the ruin probability for fixed initial surplus $u$, if the cedent's and the reinsurer's security loading are similar. If the reinsurer charges a much higher security loading, the cedent has to decrease the number of reinstatements in order to reduce the probability of ruin. This means also that more reinstatements do not necessarily improve the cedent's situation in terms of safety.

\section{Conclusion}

Excess of loss reinsurance contracts with reinstatements are widely used in practice. In the present paper we obtained both a partial integro-differential equation and an integral equation for the finite-time ruin probability. We showed that the latter can be used to express the ruin probability as the fixed point of a contractive integral operator.

This representation was then used to develop a recursive algorithm for a numerical procedure to obtain $\psi_{X L}(u, T)$. This numerical method is a valuable alternative to stochastic simulation of the risk process which allows to obtain an independent estimate of the ruin probability in this risk model. In terms of comparing computation times of the recursive 
numerical method and simulation of the risk process, it seems that (unlike in the context of dividend models, cf. Albrecher et al. [1]) the simulation method is much faster. However, as shown above, an appropriate choice of the starting function can considerably decrease the dimension of the resulting integration and this may be an advantage in certain applications (for instance when using Quasi-Monte Carlo methods to try to speed up the simulation efficiency). In the above example, a 40-dimensional integral was sufficient for the recursive method, whereas simulation of the risk process usually needs dimensions beyond 100 (as each interclaim time and claim size variable needed for the simulation of one sample path represents one dimension).

\section{Acknowledgement}

Support from the Swiss National Science Foundation Project 200021-124635/1 is gratefully acknowledged.

\section{References}

[1] H. Albrecher, R. Kainhofer, R. Tichy, Simulation methods in ruin models with nonlinear dividend barriers, Math. Comput. Simulation 62 (2003) 277-287.

[2] H. Albrecher, J. Teugels, Reinsurance: Actuarial and Financial Aspects, Wiley, Chichester, in preparation, 2011.

[3] M. Davis, Markov Models and Optimization,, Chapman \& Hall, London, 1984.

[4] A. Dassios, P. Embrechts, Martingals and insurance risk, Stochastic Models 5 (1989), 181-217.

[5] N. Furian, Excess of Loss Reinsurance with Reinstatements, Diploma Thesis, Graz University of Technology, 2007.

[6] K.T. Hess, K.D. Schmidt, Optimal premium plans for reinsurance with reinstatements, Astin Bulletin 34 (2004) 299-313.

[7] W. Hürlimann, Excess of loss reinsurance with reinstatements revisited, Astin Bulletin 35 (2005) 211-238.

[8] A.J. Mata, Pricing excess of loss reinsurance with reinstatements, Astin Bulletin 30 (2000) 349-368.

[9] T. Rolski, H. Schmidli, V. Schmidt, J. Teugels, Stochastic Processes for Insurance and Finance, Wiley, Chichester, 1999.

[10] W.C. Sandleben, Risikotheoretische Modelle für die 'Wiederauffüllung' in der Rückversicherung, Diploma Thesis, University of Karlsruhe, 1991. 
[11] B. Sundt, On excess of loss reinsurance with reinstatements, Bulletin of Swiss Actuaries (1991), 1-15.

[12] J.F. Walhin, L. Herfurth, P. de Longueville, The practical pricing of Excess of Loss treaties: actuarial, financial, economic and commercial aspects, Belgian Actuarial Bulletin 31 (2001) 1-17.

[13] J.F. Walhin, J. Paris, The effect of excess of loss reinsurance with reinstatements on the cedent's portfolio, Blätter der Deutschen Gesellschaft für Versicherungsmathematik 24 (2000) 616-627.

[14] J.F. Walhin, J. Paris, Excess of loss reinsurance with reinstatements: Premium calculation and ruin probability for the cedent, Blätter der Deutschen Gesellschaft für Versicherungsmathematik 25 (2001) 257-270.

\section{A Derivation of the partial integro-differential equation}

To prove that the solutions of $\mathscr{A} g=0$, with $\mathscr{A}$ as defined in (2) and $\mathcal{A} g=g$, with $\mathcal{A}$ given in (3) in Section 4 coincide, we differentiate the integral equation (3) w.r.t. $t$ leading to a partial integro-differential equation. We introduce the following variables:

$$
\begin{aligned}
\tilde{x} & =x-l(x, r)_{k}+\frac{c p_{0}}{m} l(x, r)_{k-1}, \\
\tilde{r} & =r+l(x, r)_{k}, \\
x^{*} & =x^{*}(u, r, t) .
\end{aligned}
$$

The partial derivative of $g((u, r), t)$ w.r.t. $t$ can be written as

$$
\begin{aligned}
\frac{\partial}{\partial t} g((u, r), t) & =\lim _{h \downarrow 0} \frac{g((u, r), t+h)-g((u, r), t)}{h} \\
& =\lim _{h \downarrow 0} \frac{1}{h}\left(\int_{0}^{t+h} \lambda \exp (-\lambda s) \int_{0}^{x^{*}} g((u+\beta s-\tilde{x}, \tilde{r}), t+h-s) d F(x) d s\right. \\
& +\int_{0}^{t+h} \lambda \exp (-\lambda s) \int_{x^{*}}^{\infty} d F(x) d s \\
& -\int_{0}^{t} \lambda \exp (-\lambda s) \int_{0}^{x^{*}} g((u+\beta s-\tilde{x}, \tilde{r}), t-s) d F(x) d s \\
& \left.-\int_{0}^{t} \lambda \exp (-\lambda s) \int_{x^{*}}^{\infty} d F(x) d s\right)
\end{aligned}
$$


Rewrite the first and second line of the above formula using the substitution $v=s-h$. This leads to the following expression for the first line:

$$
\begin{aligned}
& \int_{0}^{t+h} \lambda \exp (-\lambda s) \int_{0}^{x^{*}} g((u+\beta s-\tilde{x}, \tilde{r}), t+h-s) d F(x) d s= \\
= & \int_{0}^{t} \lambda \exp (-\lambda(v+h)) \int_{0}^{x^{*}} g((u+\beta(v+h)-\tilde{x}, \tilde{r}), t-v) d F(x) d v \\
+ & \int_{-h}^{0} \lambda \exp (-\lambda(v+h)) \int_{0}^{x^{*}} g((u+\beta(v+h)-\tilde{x}, \tilde{r}), t-v) d F(x) d v .
\end{aligned}
$$

For the second line one gets with similar considerations

$$
\begin{aligned}
\int_{0}^{t+h} \lambda \exp (-\lambda s) \int_{x^{*}}^{\infty} d F(x) d s & =\exp (-\lambda h) \int_{0}^{t} \lambda \exp (-\lambda s) \int_{x^{*}}^{\infty} d F(x) d s \\
& +\exp (-\lambda h) \int_{-h}^{0} \lambda \exp (-\lambda s) \int_{x^{*}}^{\infty} d F(x) d s
\end{aligned}
$$

The partial derivation of $g((u, r), t)$ w.r.t. $u$ can be written as:

$$
\frac{\partial}{\partial u} g((u, r), t)=\lim _{h \downarrow 0} \frac{g((u+\beta h, r), t)-g((u, r), t)}{\beta h},
$$

thus $\lim _{h \downarrow 0} \frac{1}{h}(g((u+\beta h, r), t)-g((u, r), t))=\beta \frac{\partial}{\partial u} g((u, r), t)$.

Putting together all the terms from above, one can now write the derivative of $g((u, r), t)$ w.r.t. $t$ in the following way:

$$
\begin{aligned}
\frac{\partial}{\partial t} g((u, r), t) & =\lim _{h \downarrow 0} \frac{g((u, r), t+h)-g((u, r), t)}{h} \\
& =\lim _{h \downarrow 0} \frac{1}{h}\left(\int_{0}^{t} \lambda \exp (-\lambda s) \int_{0}^{x^{*}}(g((u+\beta(s+h)-\tilde{x}, \tilde{r}), t-s)\right. \\
& -g((u+\beta s-\tilde{x}, \tilde{r}), t-s)) d F(x) d s \\
& -(1-\exp (-\lambda h))\left(\int_{0}^{t} \lambda \exp (-\lambda s) \int_{0}^{x^{*}} g((u+\beta(s+h)-\tilde{x}, \tilde{r}), t-s)\right. \\
& \left.+\int_{0}^{t} \lambda \exp (-\lambda s) \int_{x^{*}}^{\infty} d F(x) d s\right) \\
& +\int_{-h}^{0} \lambda \exp (-\lambda(s+h)) \int_{0}^{x^{*}} g((u+\beta(s+h)-\tilde{x}, \tilde{r}), t-s) d F(x) d s \\
& \left.+\exp (-\lambda h) \int_{-h}^{0} \lambda \exp (-\lambda s) \int_{x^{*}}^{\infty} d F(x) d s\right) .
\end{aligned}
$$


It is easy to see, that the following equations are fulfilled:

$$
\begin{aligned}
\beta \frac{\partial}{\partial u} g((u, r), t) & =\lim _{h \downarrow 0} \frac{1}{h}\left(\int_{0}^{t} \lambda \exp (-\lambda s) \int_{0}^{x^{*}} g((u+\beta(s+h)-\tilde{x}, \tilde{r}), t-s)\right. \\
& -g((u+\beta s-\tilde{x}, \tilde{r}), t-s) d F(x) d s), \\
\lambda g((u, r), t) & =\lim _{h \downarrow 0} \frac{1}{h}\left(\int_{0}^{t} \lambda \exp (-\lambda s) \int_{0}^{x^{*}} g((u+\beta(s+h)-\tilde{x}, \tilde{r}), t-s)\right. \\
& \left.+\int_{0}^{t} \lambda \exp (-\lambda s) \int_{x^{*}}^{\infty} d F(x) d s\right)(1-\exp (-\lambda h)), \\
\lambda \int_{x^{*}}^{\infty} d F(x) & =\lim _{h \downarrow 0} \frac{1}{h} \exp (-\lambda h) \int_{-h}^{0} \lambda \exp (-\lambda s) \int_{x^{*}}^{\infty} d F(x) d s
\end{aligned}
$$

and

$$
\lim _{h \downarrow 0} \frac{1}{h} \int_{-h}^{0} \lambda \exp (-\lambda(s+h)) \int_{0}^{x^{*}} g((u+\beta(s+h)-\tilde{x}, \tilde{r}), t-s) d F(x) d s
$$

leads to

$$
\lambda \int_{0}^{x^{*}} g((u-\tilde{x}, \tilde{r}), t) d F(x) .
$$

Therefore one can determine the differential quotient of $g((u, r), t)$ w.r.t. $t$ as

$$
\frac{\partial}{\partial t} g((u, r), t)=\beta \frac{\partial}{\partial u} g((u, r), t)+\lambda\left(\int_{0}^{x^{*}} g((u-\tilde{x}, \tilde{r}), t) d F(x)-g((u, r), t)+\int_{x^{*}}^{\infty} d F(x)\right) .
$$

The time variable in the first approach is the time until the next claim, whereas in the second approach it is the time since the last claim. Considering this fact, the solutions represented by equations (2) and (8) indeed coincide. 